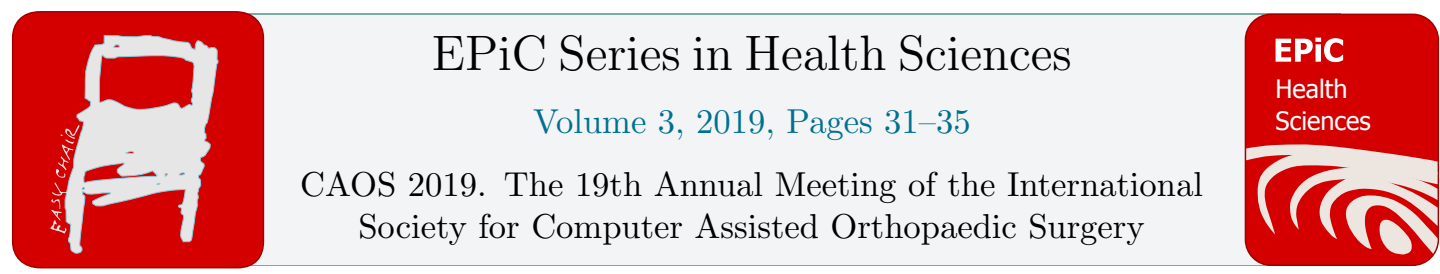

\title{
Automatic analysis of morphological parameters of the patella based on three- dimensional (3D) surface data
}

\author{
Malte Asseln*, Klaus Radermacher \\ ${ }^{1 *}$ Chair of Medical Engineering, Helmholtz Institute for Biomedical Engineering, RWTH Aachen \\ University, Aachen, 52074, Germany \\ asseln@hia.rwth-aachen.de
}

\section{Introduction}

Morphological knowledge about the patella is fundamental to understand normal and pathological knee joint mechanics and guide the design of patellar prosthesis [1]. Panni et al. [2] reported that the patella morphology can be a predisposing factor in patella instability. The congruency between the patella and femur affects the kinematics of the patellofemoral joint, contact mechanics of the patellar cartilage, and strain in the underlying bone, whereby higher stresses and strains potentially contribute to cartilage wear and anterior knee pain [3]. In patellar resurfaced knees, for example a wrong sizing can lead to overstuffing of the patellofemoral compartment, which may cause flexion deficits, patellofemoral maltracking, subluxation, and increased wear [4].

In literature there are a large number of various parameters and measurement methods described to characterize the patellar morphology $[5-7,1,8,9]$. However, the majority rely on the manual measurement of subjectively defined landmarks in medical image data or cadaveric bones using callipers. Additionally, sample sizes are often small and the number of morphological parameters is limited.

The goal of this study was to identify morphological parameters of the patella, develop a standardized and fully automatic workflow for their extraction, and to provide accurate statistical numbers for a large number of patients for improved preoperative total knee arthroplasty (TKA) planning, surgical techniques for resurfacing, and patellar prosthesis design.

\section{Materials and Methods}

An anonymized dataset of 412 knee geometries from TKA patients (gender: 248 female, 164 male) were available for the morphological analysis. The patients were consecutively scheduled for TKA, primarily in the United States. All geometries were acquired from computed tomography, segmented semi-automatically, post-processed, and stored as polygon mesh. 
Based on a literature research a total number of 8 morphological parameters of the patella were identified (Table 1) and later used for the calculation of 3 additional aspect ratios. A fully automatic workflow was developed in MATLAB (Mathworks, Inc., USA) for parameter extraction based on the three-dimensional surface data.

At first, the polygon mesh was moved to the centroid of all vertices and an anatomical coordinate system was calculated following Rainbow et al. [10]. This approach relies on principle component (PC) analysis and an iterative rotational adjustment of the PC (axes), until the first PC axis is aligned along the posterior vertical ridge. The final orthogonal anatomical coordinate system was located in the centroid of all vertices. The z-axis represented the third PC axis, pointing anteriorly. The $\mathrm{x}$-axis was aligned with the vertical ridge, pointing superiorly. The $\mathrm{y}$-axis was perpendicular to both the $\mathrm{x}$ - and $\mathrm{z}$ axis, pointing to the right.

Subsequently, the morphological parameters were calculated. The overall dimensions were calculated by taking minimum/maximum values in the directions of the coordinate axes. The referencing already revealed the medial/lateral location of the vertical ridge. On this basis and in combination with the medial/lateral extreme points the facet lengths were derived. The final workflow is illustrated in Figure 1.

The workflow was applied to all datasets and the results were stored in a database for statistical analysis. We performed a distribution analysis of the parameters using graphs, descriptive statistics, and formal statistical tests. In case of normality the parameters were described according to mean and standard deviation. Genders were treated separately, since previous showed statistical significant differences $[9,6]$.

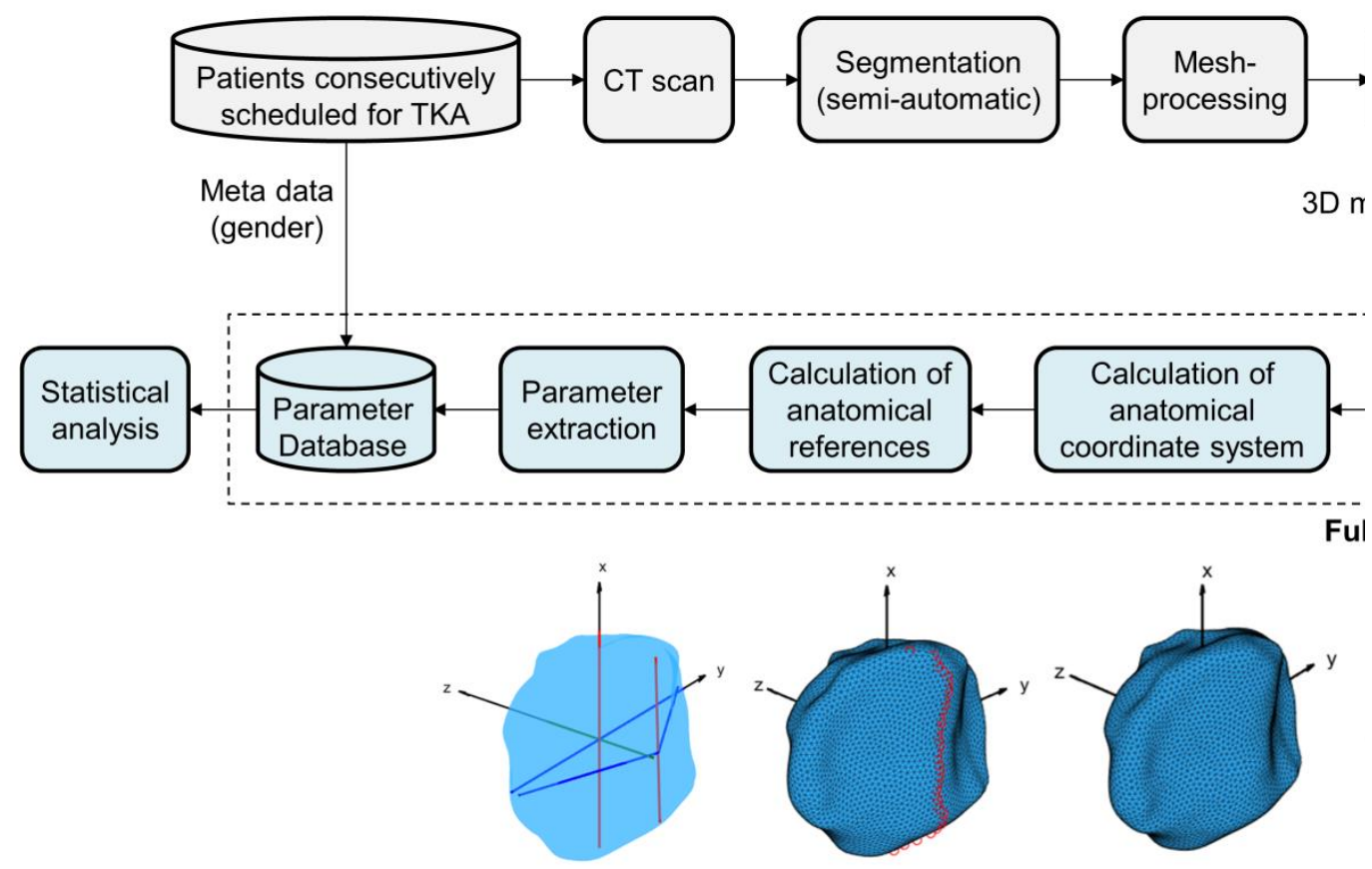

Figure 1: Visualization of the fully automatic workflow for morphological analysis of the patella. The bottom row (blue boxes) is covered within this work. 


\section{Results}

The workflow could process all 412 patellar geometries fully automatic without any algorithmic adjustment or user interaction. The processing time was in the range of $30 \mathrm{~s}$ per case resulting in $3.5 \mathrm{~h}$ for all datasets. The formal statistical tests, which are very sensitive for large sample sizes $(>100)$, indicated normality for 6 of 8 parameters in the female group and 7 of 8 parameters in the male group. The distributions of the remaining parameters were evaluated by graphs and descriptive statistics, which confirmed normality for all parameters. The mean and standard deviation are summarized in Table 1. The overall dimensions showed distinct gender-specific characteristics, whereas the mean values of the Relative Ridge Location (P04) were rather equal. Looking at the aspect ratios, the Width to Length ratio (P09) was almost 1, the Width to Ridge Length ratio (P10) ranged between 1.34 and 1.38, respectively, and the Width to Thickness ratio was about 2 (P11).

\begin{tabular}{llllrrrr}
\hline No. & \multicolumn{1}{c}{ Parameter } & Abbr. & Unit & \multicolumn{2}{c}{ Female } & \multicolumn{2}{c}{ Male } \\
& & & & Mean & SD & Mean & \multicolumn{2}{l}{ SD } \\
\hline P01 & Length & PL & {$[\mathrm{mm}]$} & 40.34 & 2.66 & 45.73 & 3.75 \\
P02 & Thickness & PT & {$[\mathrm{mm}]$} & 21.40 & 1.83 & 24.18 & 2.18 \\
P03 & Width & PW & {$[\mathrm{mm}]$} & 43.13 & 3.04 & 48.97 & 3.55 \\
P04 & Relative Ridge Location & RRL & {$[\%]$} & 0.58 & 0.04 & 0.58 & 0.03 \\
P05 & Ridge Length & RL & {$[\mathrm{mm}]$} & 31.52 & 3.87 & 37.16 & 5.27 \\
P06 & Medial Facet Length & MFL & {$[\mathrm{mm}]$} & 20.77 & 2.11 & 23.22 & 2.42 \\
P07 & Lateral Facet Length & LCL & {$[\mathrm{mm}]$} & 26.28 & 1.92 & 29.42 & 2.17 \\
P08 & Facet Angle & FA & {$\left[{ }^{\circ}\right]$} & 133.91 & 8.48 & 137.91 & 8.10 \\
P09 & Aspect Ratio (P03 / P01) & & & 1.07 & 0.07 & 1.07 & 0.08 \\
P10 & Aspect Ratio (P03 / P05) & & & 1.38 & 0.17 & 1.34 & 0.18 \\
P11 & Aspect Ratio (P03 / P02) & & & 2.02 & 0.15 & 2.03 & 0.15 \\
\hline
\end{tabular}

Table 1: Morphological parameters of the patella and their statistical results for female and male.

\section{Discussion}

We developed a standardized and fully automatic workflow to extract 8 morphological parameters of the patella. The results of the statistical analysis were in very good agreement with the literature. For example, Yoo et al. [1] reported $40.0 \pm 2.6 \mathrm{~mm}$ (female) and $45.6 \pm 3.0 \mathrm{~mm}$ (male) for the whole longitudinal length (P01), whereas we found $40.34 \pm 2.66 \mathrm{~mm}$ (female) and $45.73 \pm 3.75 \mathrm{~mm}$ (male). The other overall dimensions were in the same good range. Looking at the Medial Facet Length (P06) and Lateral Facet Length (P07), the lateral was larger than the medial facet for both genders. This has been confirmed by Fucentese et al. [8], who reported pathological values of the lateral facet of $25.55 \pm$ $2.31 \mathrm{~mm}$ and $18.73 \pm 3.57 \mathrm{~mm}$ for the medial facet, respectively. However, they did not consider gender-specific differences. Takahashi et al. [11] investigated the patellar facet angle (P08) and reported an average angle in all patients and knees of $133 \pm 6^{\circ}$. Our Facet Angle (P08) was $133.91 \pm 8.48^{\circ}$ (female) and $137.91 \pm 8.10^{\circ}$ (male) . 
The numbers clearly demonstrated gender-specific differences, however, it is still unknown whether these are sexual dimorphism or can be eliminated by an adequate scaling. The Aspect Ratio (P10) indicates that patellar prostheses aimed for an adequate reconstruction of the articulating portion should be oval in shape as opposed to spherical and approximately 1.3 times as wide as height. The Relative Ridge Location (P05) showed that the patella component should be medialized by about $8 \%$ with respect to the mediolateral center or the component design should have an appropriate offset of $58 \%$ from the lateral border. Extensive statistical analysis on gender-specific differences, their normalization, and the analysis of healthy cases is part of future work.

\section{Disclosures}

This work has been supported in parts by ConforMIS, Inc., Billerica, USA.

\section{References}

[1] Yoo JH, Yi SR, Kim JH. The geometry of patella and patellar tendon measured on knee MRI. Surgical and radiologic anatomy SRA 2007;29:623-8.

[2] Panni AS, Cerciello S, Maffulli N, Di Cesare M, Servien E, Neyret P. Patellar shape can be a predisposing factor in patellar instability. Knee surgery, sports traumatology, arthroscopy official journal of the ESSKA 2011;19:663-70.

[3] Fitzpatrick CK, Clary CW, Laz PJ, Rullkoetter PJ. Relative contributions of design, alignment, and loading variability in knee replacement mechanics. Journal of orthopaedic research official publication of the Orthopaedic Research Society 2012;30:2015-24.

[4] Ghosh KM, Merican AM, Iranpour F, Deehan DJ, Amis AA. The effect of overstuffing the patellofemoral joint on the extensor retinaculum of the knee. Knee surgery, sports traumatology, arthroscopy official journal of the ESSKA 2009;17:1211-6.

[5] Olateju OI, Philander I, Bidmos MA. Morphometric analysis of the patella and patellar ligament of South Africans of European ancestry. SAJS 2013;109:1-6.

[6] Peckmann TR, Meek S, Dilkie N, Rozendaal A. Determination of sex from the patella in a contemporary Spanish population. Journal of forensic and legal medicine 2016;44:84-91.

[7] Stäubli HU, Dürrenmatt U, Porcellini B, Rauschning W. Anatomy and surface geometry of the patellofemoral joint in the axial plane. The Journal of bone and joint surgery. British volume 1999;81:452-8.

[8] Fucentese SF, Roll A von, Koch PP, Epari DR, Fuchs B, Schottle PB. The patella morphology in trochlear dysplasia--a comparative MRI study. The Knee 2006;13:145-50.

[9] Mahfouz M, Badawi A, Merkl B, Fatah EEA, Pritchard E, Kesler K et al. Patella sex determination by 3D statistical shape models and nonlinear classifiers. Forensic science international 2007;173:161-70.

[10] Rainbow MJ, Miranda DL, Cheung RTH, Schwartz JB, Crisco JJ, Davis IS et al. Automatic Determination of an Anatomical Coordinate System for a Three-Dimensional Model of the Human Patella. Journal of biomechanics 2013;46:2093-6. 
[11] Takahashi A, Sano H, Ohnuma M, Kashiwaba M, Chiba D, Kamimura M et al. Patellar morphology and femoral component geometry influence patellofemoral contact stress in total knee arthroplasty without patellar resurfacing. Knee surgery, sports traumatology, arthroscopy official journal of the ESSKA 2012;20:1787-95. 